\title{
Do Not Forget Proximal Hamstring Avulsions
}

\section{Diego Edwards $^{1 *}$, Miguel Carrasco ${ }^{1}$ and Consuelo Carrasco ${ }^{2}$}

${ }^{1}$ Clinica Alemana, Universidad del Desarrollo, Sports Medicine, Santiago, Chile

${ }^{2}$ Faculty of Medicine, Universidad Los Andes, Santiago, Chile

*Corresponding Author: Diego Edwards, Traumatology and Orthopaedic Surgery Department, Clinica Alemana, Universidad del Desarrollo, Sports Medicine, Santiago,

Chile.
Received: August 27, 2021

Published: September 23, 2021

(C) All rights are reserved by Diego Edwards., et al.
Proximal hamstring avulsions (PHA) are rare lesions that can cause permanent functional alterations and a very high risk of retear $(12 \%$ to $31 \%)$ [1]. They are present in $9-12 \%$ of all lesions in this muscle group [2] and most often occur in athletes who require rapid acceleration (running, hurdling) or "ballistic" movements (skiing, skating, weight lifting) $[3,4]$.

Proximal hamstrings comprise 3 tendons: the semitendinosus, the long head of the biceps femori and the semimembranosus (the latter 2, merged at their origin by the conjoined tendon). The mechanism of injury corresponds to eccentric contraction of the $\mathrm{PH}$ when trying to resist a fall, with the hip in flexion and the knee in extension. Acute pain is usually reported in the posterior thigh, sometimes accompanied by an audible pop and in some occasions with numbness and tingling in the sciatic nerve distribution [5].

On physical examination the patient may have an ecchymosis in the posterior thigh, with an altered gait, avoiding simultaneous flexion of the hip and extension of the knee ("hurdler's position") [5]. It is also important to assess the neurological state in the sciatic nerve territory. Among the most common tests are:

- Bowstring sign: Absence of palpable tension in the distal part of the hamstring with the patient prone and the knee flexed $90^{\circ}$.

- Popliteal angle: In the supine position, with the hip and knee flexed $90^{\circ}$, the knee is gently extended; then the knee flexion is measured and compared with the unaffected side (affected side having a greater angle).
- Active shoe removal sign: Patients will have more difficulty removing the shoe on the affected side.

- Hip extension test: In the prone position, the patient may not be able to extend the hip on the affected side.

Imaging begins with a plain radiography of the pelvis and affected hip which is most often negative, unless an avulsion fracture from the ischial tuberosity occurs in skeletally immature patients. Ultrasonography is readily available and may provide with valuable information, although the MRI is considered the gold standard since it can differentiate between partial or complete avulsion, acute and chronic injuries, number of tendons involved, tendon retraction and muscle quality (fatty infiltration and atrophy). MRI could also show structural alteration of the sciatic nerve or some compression due to a mass effect [6].

Treatment for PHA may be conservative or surgical, depending on the patient's age, level of activity and imaging characteristics of the lesion. In general, the current studies published are of low quality, with case series that include only patients treated surgically $[7,8]$ and only a few comparative studies in which most of the patients included were operated on [9], therefore surgical indications may be debatable. However, the current recommendation based on published studies is to reserve surgery for young, active patients with either a 2-tendon tear/avulsion with more than $2 \mathrm{~cm}$ retraction or those with complete 3 -tendon tears $[4,10]$ or conservative treatment failure, whether due to major functional impairment, pain, or sciatic nerve involvement. Acute repair $(<4$ weeks) 
has better functional results, greater sports return and fewer complications than late surgeries $[3,11]$.

Surgical treatment is usually performed openly, although there are successful reports using endoscopic and mini open techniques, as shown by the studies of Factor [12], Domb [13] and Laskovski., et al [14]. However, the current recommendation is to implement it in an open manner, especially in groups that do not have extensive experience in this field. Fixation has also evolved over time, from old techniques with transosseous fixation to now repairing with anchors. The number of anchors is also a subject of debate, with groups using 3 anchors [5] and others with 5 anchors [15].

Rehabilitation protocols usually include an initial non-weight period, avoiding knee extension over 30 degrees (some groups recommend higher degrees of knee block) and hip extension to protect the fixation. The return to normal activities of daily living normally occurs at 2 - 3 months, with a progressive return to sports activities between 6 - 9 months.

\section{Conclusion}

PHA are rare and difficult lesions to manage, which can leave serious functional sequelae. A high level of suspicion is mandatory, MRI should be part of the study and referral may be considered for eventual surgical fixation in some cases. An active return to sports is achievable, which usually occurs between 6 - 9 months.

\section{Bibliography}

1. Croisier JL. "Factors associated with recurrent hamstring injuries". Sports Medicine 34.10 (2004): 681-695.

2. Koulouris G and Connell D. "Evaluation of the hamstring muscle complex following acute injury". Skeletal Radiology 32 (2003): 582-589.

3. Ali K and Leland JM. "Hamstring strains and tears in the athlete”. Clinical Journal of Sport Medicine 31.2 (2012): 263-272.

4. Cohen S and Bradley J. "Acute proximal hamstring rupture". Journal of the American Academy of Orthopaedic Surgeons 15.6 (2007): 350-355.

5. Bertiche P., et al. "Proximal hamstring tendon avulsion: state of the art". Journal of ISAKOS 6 (2021): 237-246.
6. Koulouris G and Connell D. "Hamstring muscle complex: an imaging review". Radiographics 25 (2005): 571-586.

7. Bodendorfer BM., et al. "Outcomes after operative and Nonoperative treatment of proximal hamstring Avulsions: a systematic review and meta-analysis". The American Journal of Sports Medicine (2017): 363546517732526.

8. Cohen SB., et al. "Functional results and outcomes after repair of proximal hamstring avulsions". American Journal of Sports Medicine 40 (2012): 2092-2098.

9. Pihl E., et al. "Patient-reported outcomes after surgical and non-surgical treatment of proximal hamstring avulsions in middle aged patients". BMJ Open Sport and Exercise Medicine 5 (2019): e000511.

10. Harris JD., et al. "Treatment of proximal hamstring ruptures $\mathrm{dA}$ systematic review". International Journal of Sports Medicine 32 (2011): 490-495.

11. Sallay PI., et al. "Subjective and functional outcomes following surgical repair of complete ruptures of the proximal hamstring complex". Orthopedics 31 (2008): 1-7.

12. Factor., et al. "Combined endoscopic and mini-open repair of chronic complete proximal hamstring tendon avulsion: a novel approach and short-term outcomes". Journal of Hip Preservation Surgery 7.4 (2021): 721-727.

13. Domb BG., et al. "Endoscopic repair of proximal hamstring avulsion". Arthroscopy Techniques 2 (2013): e35-39.

14. Laskovski JR., et al. "Endoscopic proximal hamstring repair and ischial bursectomy using modified portal placement and patient positioning". Arthroscopy Techniques 7 (2018): e10711078.

15. Moatshe., et al. "Repair of Proximal Hamstring Tears: A Surgical Technique". Arthroscopy Techniques 6.2 (2017): e311-e317.

\section{Volume 4 Issue 10 October 2021 (C) All rights are reserved by Diego Edwards., et al.}

